\title{
Reflexiones sobre la Ley 34/2003 (3). Valoración de los capítulos relativos a raquis, extremidades superiores e inferiores.
}

\author{
Commentaries on the Spanish Law 34/2003 (3). Evaluation \\ of the chapters concerning spinal column, upper and lower \\ extremities.
}

PM. Garamendi ${ }^{1}$

\begin{abstract}
RESUMEN
El 5 de noviembre de 2003 se publicó la nueva Ley 34/2003 de modificación y adaptación a la normativa comunitaria de la legislación de seguros privados que incluyó la modificación de la tabla VI de baremo de indemnización de secuelas definitivas de la Ley 30/1995 de ordenación y supervisión de seguros privados. En nuestro país, el citado baremo ha constituido y constituye la herramienta aplicada en el contexto judicial para la indemnización económica de las lesiones producidas con motivo de accidentes de tráfico. La anterior redacción del citado baremo ha sido motivo de enconadas controversias en los distintos foros de debate relacionados con la Valoración del Daño Corporal desde su implantación. Sin negar la necesidad de algún instrumento de valoración común que colaborase a evitar la situación de caos aparente existente en nuestro país hasta la implantación del baremo de tráfico, han sido múltiples las críticas expresadas sobre su fundamento científico, validez y redacción final. El presente trabajo intenta adelantar algunas críticas y propuestas de mejora sobre la nueva redacción del baremo, centrándose en las modificaciones incluidas en el capítulo de raquis, extremidades superiores e inferiores en el aparato locomotor.
\end{abstract}

Palabras clave: accidentes de tráfico; baremo; extremidades superiores; extremidades inferiores; ley 34/2003; traumatología; raquis; valoración del daño corporal.

\section{ABSTRACT}

On November 52003 the new Law 34/2003 was published. This law entails the modification and adaptation to the community normative concerning legislation of private insurance that included the modification of the chart $\mathrm{VI}$ of scale of compensation of definitive sequels of the Law 30/I 995 of ordination and supervision of private insurance. In our country, the mentioned scale has constituted and constitutes the tool applied in the judicial context for economic compensation of the lesions taken place in traffic accidents. The previous writing of the mentioned scale has been the cause of inflamed controversies in the different debate forums related to the Valuation of the Corporal Damage from its installation. Without denying the necessity of some instrument of common valuation that collaborates to avoid the situation of existing apparent chaos in our country until the installation of the traffic scale, they have been multiple the criticisms expressed on their scientific basis, validity and final writing. The present paper tries to advance some criticisms and proposals of improvement on the new writing of the scale, being centered on the modifications included in the chapter of spinal column, upper and lower extremities in the locomotor aparatus.

Key words: traffic accidents; guides for the assessment; upper extremities; lower extremities; Spanish Law 34/2003; Traumatology; spinal column; permanent impairment assessment.

Correspondencia: Pedro Manuel Garamendi González. Servicio de Clínica Médico Forense. c/ Buenos Aires, nº 6 , planta 4. Bilbao 48001. garamendi.pm@aju.ej-gv.es.

1 Médico forense. Especialista en Medicina Legal y Forense. Servicio de Clínica Médico Forense. IVML. Vizcaya. 


\section{INTRODUCCIÓN:}

El 5 de noviembre de 2003 se publicó la nueva Ley 34/2003 de modificación y adaptación a la normativa comunitaria de la legislación de seguros privados [I] que incluyó la modificación de la tabla VI de baremo de indemnización de secuelas definitivas de la Ley 30/1995 de ordenación y supervisión de seguros privados [2]. En nuestro país, el citado baremo ha constituido y constituye la herramienta aplicada en el contexto judicial para la indemnización económica de las lesiones producidas con motivo de accidentes de tráfico.

La anterior redacción del baremo, incluida en la tabla VI de la Ley 30/1995, fue motivo de enconada controversia desde su implantación en nuestro país. Su implantación fue una respuesta lógica a la situación de caos indemnizatorio por secuelas en accidentes de tráfico existente en nuestro país antes de su desarrollo [3]. Tanto desde los foros médico legales, como desde las compañías aseguradoras y desde los propios medios judiciales [3], la medida fue aplaudida como un medio de unificación y armonización de una situación difícilmente sostenible antes de 1995 en relación con la indemnización de daños personales en accidentes de tráfico. Sin embargo, pese a ello, en los 8 años de vida efectiva del baremo, este ha sido objeto de múltiples críticas desde los distintos colectivos vinculados a su uso habitual. Las críticas principales fueron centradas en su redacción imprecisa, fundamento científico altamente dudoso y terminología no actualizada al actual estado de conocimientos en ciencias biomédicas [4].

La nueva redacción de la tabla-baremo de indemnización de daños personales ha sido presentada por sus autores en distintos foros nacionales como una mejora significativa en relación con su precedente. Se han argumentado por parte de los mismos mejoras en la ordenación y jerarquización de las secuelas, en el fundamento científico de la cuantificación del daño corporal, en el equilibrio en las cuantías de indemnización entre distintos capítulos del baremo y en el ajuste terminológico a la terminología médica más actualizada $[5,6]$. Sin embargo, pese a reconocer las mejoras evidentes en la redacción de la nueva tabla baremo y los esfuerzos por parte del grupo de trabajo auspiciado por UNESPA, el texto final presentado a las Cámaras legislativas en junio de 2003 y el texto definitivo publicado en el BOE en noviembre de 2003 están aún lejos de constituir un instrumento idóneo de indemnización de daños personales [7,8].

El presente trabajo se centra en el análisis de los cambios efectuados en los capítulos relativos a raquis, extremidades inferiores y superiores del nuevo baremo. Este análisis está orientado a valorar si la redacción del nuevo baremo cumple en los capítulos indicados los elementos definitorios de un baremo de indemnización con un adecuado fundamento científico planteados en un trabajo precedente [9].

Los elementos definitorios señalados previamente han sido:

I. Definición clara del objetivo de valoración de los daños personales. Concreción en la valoración de daños estrictamente funcionales (físicos y psíquicos), diferenciándola de la valoración de daños morales u otras modalidades de daños personal.

2. Fundamento científico adecuado.

3. Marco abierto y no excluyente de asignación de estados clínicos que permitan la inclusión de todo tipo de estados clínicos con repercusión funcional real.

4. Redacción clara con adecuación terminológica a los términos manejados en la literatura médica actualizada.

5. Adecuada consistencia interna o validez en sus sistemas de asignación de grados de gravedad. 
6. Congruencia con otros baremos ya existentes y validados, con estudios válidos que demuestren la eficacia de los métodos de cuantificación como predictores de un menoscabo funcional proporcionado y con las recomendaciones de las comisiones de expertos, cuando estos se hallen disponibles.

Los principios indicados, exigibles a toda prueba biomédica, resultan esenciales para obtener dos objetivos principales en la aplicación práctica de un baremo de daños corporales:

I. Una correcta asignación de gravedad relativa al estado clínico residual de un lesionado basada en un principio de proporcionalidad.

2. Una correcta reproducibilidad de los resultados en el caso de que dos peritos expertos evalúen de forma independiente al mismo lesionado.

\section{DEFINICIÓN DEL OBJETIVO DE VALORACIÓN DE LOS DAÑOS PERSONALES EN EL BAREMO ESPAÑOL:}

Tanto el baremo de indemnización de daños personales incluido en la Ley 30/I 995, como su nueva redacción en la Ley 34/2003, presentan un obstáculo fundamental para su adecuada validación técnica médico-legal o su comparación con otros métodos de valoración de daños personales utilizados en otros países. Este obstáculo reside en el hecho de que el sistema de puntuación de gravedad de daños personales incluye, según la norma $1^{\circ}$, punto $7^{\circ}$ del anexo de la ley 30/1995, no modificada por la Ley 34/2003 [2], tanto la reparación de los "daños corporales" como la de los "daños morales", indicando además que la reparación de los daños morales será uniforme para todos los estados secuelares, con independencia de su origen o de la repercusión funcional final.

Sin duda, ésta debe ser la razón por la cual todas las categorías de secuelas definitivas del baremo presentan la peculiaridades de iniciar todos sus horquillas de puntuaciones en valores de al menos I punto. Médicamente, todos los sistemas de valoración de daños personales estrictamente funcionales parten de la asunción de que un estado secuelar puede existir realmente y no producir alteraciones funcionales significativas en el sujeto, pudiendo corresponder con estados de deterioro funcional equiparables con un $0 \%$ de menoscabo. Esta es la filosofía de baremos como las tablas AMA (American Medical Association) o de los sistemas indemnizatorios en países anglosajones $[\mathrm{IO}, \mathrm{I} \mid \mathrm{I}$.

Esta peculiaridad del baremo español, útil para el legislador con el fin de unificar la reparación de los daños morales y unificar la discrecionalidad jurisdiccional en un sistema de reparación de daños personales abierto $[3,9]$, resulta un grave obstáculo para la valoración por parte de médicos valoradores y para la validación del sistema en comparación con otros métodos de cuantificación del menoscabo previos. De una parte, como se ha indicado, los médicos legistas y valoradores tal vez no sean los profesionales más capacitados técnicamente para valorar los daños morales, sea de forma directa o indirecta, y hasta cierto punto el sistema de baremo español, cuando es aplicado estrictamente por un médico, puede suponer una intromisión en una labor que debiera estar reservada a los juristas, como es la de cuantificar daños morales, de una entidad mucho menos individualizada y abierta que el posible menoscabo funcional desde un punto de vista médico legal estricto. De otra parte, el sistema de indemnización unificado de daños morales y funcionales supone también un obstáculo para la comparación de la tabla baremo española con otros sistemas de cuantificación de daños funcionales o de indemnización de estos, como el basado en las tablas AMA y sus variantes, que parten de unos principios teóricos diversos, en los que sólo se pretende la cuantificación del menoscabo o daño estrictamente funcional; en ningún caso, daños morales. 


\section{FUNDAMENTO CIENTÍFICO DEL BAREMO:}

El sistema de baremo de indemnización de daños personales español, al igual que otros baremos previos $[10,11,12,13]$, presenta la característica común de partir de las conclusiones de reuniones de expertos. Sin embargo, pocos de los sistemas de baremo existentes presentan un origen basado en evidencias científicas contrastadas previas. De hecho, la mayoría de estudios realizados para valorar la utilidad relativa de los sistemas de valoración de la repercusión funcional de los daños corporales no han influido en las redacciones de los distintos sistemas existentes de forma significativa.

En el baremo español, además, se unen una serie de características definidas por sus autores como "virtudes" fundamentales cuando, analizadas de forma rigurosa, no puede por menos que valorarse las mismas como posibles errores metodológicos sustanciales $[5,6]$. Destacan entre estas supuestas virtudes [5] el hecho de que se planteen parámetros de valoración supuestamente objetivos, como los arcos de movilidad, sin especificar aspectos básicos como la fuente de investigación en la que se basan sus afirmaciones de normalidad. En este sentido, el análisis superficial de las tablas anexas I al 4 pone de manifiesto la evidente discrepancia con los parámetros de "normalidad" de un supuesto parámetro objetivo como es el arco de movilidad. De la misma forma, tampoco se especifican aspectos esenciales de cualquier cuantificación de arcos de movilidad, como las condiciones en las que el arco es medido (obsérvese, en este sentido, las diferencias entre los distintos métodos descritos en la movilidad del hombro, como los de la escala de Constant [14] o el texto de Kapandji [15]). Igualmente, no se especifica si los arcos de movilidad deben ser medidos funcionalmente como arcos activos o pasivos o como hallazgos de exploración física o radiográfica, aspectos sustanciales que alteran claramente los resultados [15]. Finalmente, tampoco se considera el hecho de que los arcos de movilidad normales se modifican de forma individualizada con factores tan simples como la edad, el sexo o el entrenamiento previo.

En la misma línea de argumentación, los autores del baremo consideran un éxito del mismo el hecho de que síntomas, signos, síndromes, estados funcionales y arcos de movimiento se agrupen indistintamente en los distintos capítulos del baremo como parámetros de indemnización sin guías específicas o árboles de decisión bien definidos [5]. Esta interpretación resulta un tanto dudosa. De hecho, tablas de uso generalizado a nivel internacional, como las tablas AMA, ya han considerado esta circunstancia $y$, a diferencia del grupo de trabajo de UNESPA, han incluido en su redacción árboles de decisión simples en la elección de los métodos de cuantificación del menoscabo funcional. Sin embargo, en honor a la verdad, es necesario reconocer el enorme avance que supone dentro de la nueva redacción del baremo la inclusión de la norma $2^{\mathrm{a}}$ de carácter general [I], que de un forma genérica incluye un intento de organización en el sistema de cuantificación del daño corporal. No obstante, tal vez hubiese sido conveniente ampliar esta norma con la inclusión de árboles de decisión más prácticos, en la línea de las tablas AMA [I0]. Un ejemplo de ello se encuentra en las EBD de grado 3 y 5 de columna cervical (tabla 2) que permiten la combinación de resultados con patrones específicos de radiculopatía, a diferencia de los grados 1 y 2 que no permiten valoraciones combinadas 0 las EBD (estimaciones basadas en el diagnóstico) por lesión discal que deben ser combinadas con limitaciones de movilidad y alteraciones neurológicas.

Finalmente, puede destacarse en el capítulo de raquis y extremidades superiores e inferiores el hecho de que algunas de las críticas a baremos previos, como las tablas AMA, no han sido 
tomados en consideración de forma congruente con el actual estado de la cuestión en valoración de resultados en patología traumatológica $[4,16]$. En estudios previos, se han puesto en tela de juicio, entre otros, los métodos de cuantificación de los resultados basados en arcos de movimiento en segmentos anatómicos como el tobillo y pie [17], columna vertebral $[18,19,20]$ y la función de la mano [2 I] al considerar que no reflejan de forma clara el grado de menoscabo funcional real provocado en la función global del sujeto ni sirven como parámetros de valoración del grado de discapacidad asociado. En este sentido, el baremo español reitera algunos de los defectos de tablas previas, como la de Melennec [12] o versiones previas de las tablas AMA, basados en bienintencionados esfuerzos de consenso entre expertos pero que las evidencias científicas han demostrado no tan útiles como se planteaban originalmente en la práctica.

\section{EL BAREMO ESPAÑOL COMO MARCO ABIERTO Y NO EXCLUYENTE DE ASIGNACIÓN DE ESTADOS CLÍ- NICOS QUE PERMITAN LA INCLUSIÓN DE TODO TIPO DE ESTADOS CLÍNICOS CON REPERCUSIÓN FUNCIONAL REAL:}

Suponer que un baremo puede incluir una lista de secuelas y de estados patológicos absolutamente integral en la que todos los posibles estados patológicos queden reflejados y cuantificados resulta ilusorio. Posiblemente, ni siquiera partir del listado integral de estados patológicos recogidos en la clasificación ICD- 10 (Clasificación Internacional de Enfermedades) [22] permitiese reflejar todos los posibles estados patológicos y, sobre todo, sería difícil reflejar en la valoración cuantitativa de los mismos la complejidad funcional inherente a algunos de ellos y la individualización necesaria de cada caso para cuantificar de forma precisa el menoscabo funcional en toda su complejidad. Resulta evidente que el baremo de la Ley 34/2003 no pretende ni puede pretender tal empresa.

Pese a que la redacción del baremo no especifica norma alguna sobre la forma en la que deben valorarse las secuelas definitivas que no están recogidas en la redacción, la práctica habitual en las clínicas médico forenses es la de buscar analogías, a menudo remotas, entre las categorías existentes en el baremo y ciertas patologías no contempladas en el mismo con el fin de asegurar que las mismas sean de alguna manera valoradas e indemnizadas. Esta práctica habitual, a la que nos hemos acostumbrado de forma necesaria, en realidad refleja uno de los defectos estructurales del baremo. Tal vez, la decisión de elegir un sistema de agrupación inconexa de síndromes con signos, síntomas y alteraciones estructurales, ya comentada anteriormente, colabore en parte a este defecto.

Este problema ha intentado ser solventado de alguna manera en otros baremos, como las tablas AMA, mediante las llamadas EBD o estimaciones basadas en el diagnóstico en oposición a las categorías diagnósticas cerradas. La obligación de elegir entre ambos sistemas de valoración facilita la posibilidad de, a un tiempo, asegurar estimaciones tasadas de estados patológicos habituales y solventar de forma satisfactoria las estimaciones de estados patológicos complejos o infrecuentes no reflejados entre las categorías diagnósticas tasadas. Una solución similar hubiese sido conveniente en la nueva redacción del baremo español.

\section{REDACCIÓN PRECISA DEL BAREMO CON ADECUACIÓN TERMINOLÓGICA A LOS TÉRMINOS MANEJA- DOS EN LA LITERATURA MÉDICA ACTUALIZADA:}

Este es quizás uno de los capítulos de la redacción del nuevo baremo que han sufrido una mejora más significativa, en lo relativo a los capítulos de lesiones traumatológicas, si bien no tanta en otros capítulos como los del daño psiquiátrico. Algunas incongruencias terminológicas y de adscrip- 
ción proporcionada de puntuaciones de la antigua redacción han mejorado de forma sustancial en lo relativo a su claridad de redacción (como la del infaustamente famoso antiguo capítulo de la movilidad del codo). Sin embargo, se sigue echando en falta una guía acompañante al baremo que establezca oficialmente los parámetros estándar bajo los cuales deben tomarse ciertas medidas descritas en el baremo y en algunos casos los propios criterios cuantitativos de arcos de normalidad, como en la columna cervical. Las tablas I al 4 reflejan algunas diferencias entre distintos sistemas de valoración, por ejemplo, de los arcos de movimiento. En aras de una adecuada reproducibilidad de resultados, una guía que estableciese las condiciones en las que el baremo pretende que se establezcan las mediciones sería conveniente.

\section{CONSISTENCIA INTERNA O VALIDEZ EN EL SISTEMA DE ASIGNACIÓN DE GRADOS DE GRAVEDAD EN EL BAREMO:}

Este es quizás uno de los problemas principales con los que el baremo español se enfrenta en cuanto a su carácter científico. La consistencia interna o validez es un parámetro que se debe solicitar a toda prueba biomédica que pretenda valorar y cuantificar items distintos. Esta consistencia interna permite decidir que realmente la prueba mide lo que dice medir y lo hace de forma proporcionada, asignando a cada item la importancia relativa que realmente tiene en el conjunto de la economía funcional analizada. Esta consistencia se debe valorar tanto a nivel longitudinal como transversal. La consistencia longitudinal se referirá a la jerarquía de puntuaciones establecida en cada capítulo y la consistencia transversal se referirá a la comparación entre secuelas con análogas puntuaciones relativas procedentes de distintos capítulos. Los ejemplos de inconsistencia interna longitudinal y transversal del baremo español, pese a sus mejoras, son múltiples.

Respecto de la consistencia longitudinal, se aprecia una mejora significativa entre las categorías del nuevo baremo en cada uno de los capítulos frente a la consistencia del anterior baremo. Existe una valoración mucho más proporcionada, con valoraciones progresivas más ajustadas entre secuelas con carácter de gravedad progresivo. Sin embargo, persisten errores notables que parten de problemas metodológicos internos del propio baremo. Un ejemplo simple se puede derivar de la tabla 3 anexa relativa a los arcos de movilidad en la extremidad superior. En el capítulo de hombro, el antiguo baremo no incluía normas de interpretación y el nuevo sólo establece la norma de que la suma de secuelas concurrentes no debe superar el valor de amputación de la extremidad superior; norma, por otra parte razonable y bienvenida por parte de los médicos encargados de aplicar el baremo. Sin embargo, si se observa el valor obtenido por el sumatorio de puntos por limitación de movilidad del segmento del hombro, se encuentra que la suma, tanto en la antigua como en la nueva redacción del baremo, excede los valores máximos otorgados para una anquilosis de hombro en posición no funcional, es decir, la peor de las circunstancias en caso de limitación máxima de movilidad por causa anatómica. Este sumatorio, por otra parte, en el nuevo baremo duplica el valor máximo admitido por anquilosis en posición no funcional y todo ello sin superar el valor máximo admitido de amputación unilateral del hombro. Si se observa en la misma tabla los valores obtenidos aplicando la tabla AMA para el mismo segmento de movimiento se aprecia la exquisita consistencia interna longitudinal en la construcción del sistema de items de esta tabla baremo.

Respecto a su consistencia interna transversal, resulta incomprensible la valoración coincidente de ciertas secuelas correspondientes a capítulos del baremo diversos. Un ejemplo claro es del 
trastorno por estrés postraumático (I-3 puntos) que merece una puntuación de gravedad relativa análoga a la de material de osteosíntesis en el carpo ( I-3 puntos). Ambas circunstancias clínicas no merecen comparación en sí mismas si se considera en toda su potencial gravedad y en todo su carácter limitante un cuadro de estrés postraumático crónico. En la misma línea, se consideran puntuaciones dispares en casos como una parálisis de nervio subescapular (6- I0 puntos) y en la limitación de movilidad por rotación interna ( I-5 puntos). Sin embargo, los músculos rotadores internos del hombro inervados por el nervio subescapular son el músculo subescapular y el redondo mayor $y$, los otros dos músculos rotadores, el dorsal ancho y pectoral mayor, son inervados por el nervio toracodorsal y por los nervios pectorales. Ello condiciona que la limitación funcional por debilidad de rotación interna sea relativamente bien tolerada en caso de lesión aislada del nervio subescapular [23]. Es destacable el hecho de que la tabla AMA otorga a la parálisis del subescapular una puntuación de sólo un $2 \%$ de menoscabo y a la limitación conjunta de la rotación interna y externa un $6 \%$ de menoscabo, en una proporcionalidad más ajustada. En el mismo sentido, la paresia del nervio circunflejo (2- 10 puntos) que produciría una debilidad de abducción del hombro, pero no anulación de función del deltoides, y que a menudo es bien tolerada por los pacientes afectos [4], se equipara con el margen máximo de limitación anatómica de abducción en el arco de $45^{\circ}$ a $90^{\circ}$ (5- 10 puntos), que puede suponer un factor de limitación aún más severo para el paciente.

\section{CONGRUENCIA CON OTROS BAREMOS YA EXISTENTES Y VALIDADOS, CON ESTUDIOS VÁLIDOS QUE DEMUESTREN LA EFICACIA DE LOS MÉTODOS DE CUANTIFICACIÓN COMO PREDICTORES DE UN MENOSCABO FUNCIONAL PROPORCIONADO Y CON LAS RECOMENDACIONES DE LOS COMITÉS DE EXPERTOS, CUANDO ESTOS SE HALLEN DISPONIBLES:}

Las tablas I al 4 anexas al texto se hacen eco de las evidentes diferencias de valoración de gravedad relativa del nuevo baremo español y del antiguo con la versión española oficial de las tablas AMA (RD 197।/I 999 [ I 0]) y con los indicadores de normalidad considerados en textos clásicos de fisiología articular [15] o en manuales de exploración de uso generalizado por parte de la comunidad médica [24].

Respecto de los posibles estudios de evidencias de eficacia de los marcadores utilizados como parámetros de cuantificación del menoscabo, ya se ha apuntado en líneas precedentes la falta de consideración de estudios validados internacionales que indican la limitada utilidad de algunos de los parámetros utilizados como indicadores de menoscabo en este nuevo baremo. En el mismo sentido, algunas recomendaciones de grupos de expertos internacionales, disponibles en la bibliografía médica nacional e internacional, tampoco parecen haber sido tomados en consideración en la globalidad de la redacción del nuevo baremo $[4,16]$.

\section{CONCLUSIONES:}

I. Resulta innegable el hecho de que la nueva versión de la tabla-baremo de reparación de daños personales por accidentes de tráfico incluida en la ley 34/2003 presenta mejoras ciertas en la valoración de los daños por causa traumatológica frente a la redacción de la versión precedente, incluida en la Ley 30/1995. Sin embargo, el análisis del nuevo baremo revela una serie de deficiencias importantes que lo alejan de ser un instrumento ideal de valoración de daños personales por causa traumatológica. 
2. Respecto de la definición del objetivo de cuantificación del baremo, sería conveniente que en ediciones posteriores del mismo se eliminase la actual asociación de daños morales y menoscabo funcional, con el fin de evitar errores de interpretación técnica en el trabajo de valoración del daño corporal por parte de los médicos legistas y valoradores.

3. El fundamento científico del sistema de cuantificación de los daños corporales en los capítulos de raquis y extremidades es dudoso y exigiría una revisión crítica por parte del observatorio de seguimiento del nuevo baremo con inclusión de las fuentes científicas en las que basan sus estimaciones de normalidad y de proporcionalidad del menoscabo estimado.

4. El carácter abierto y proporcionado del nuevo baremo en los capítulos de raquis y extremidades es limitado y exigiría una revisión de la metodología de estructuración de las secuelas en los capítulos de raquis y extremidades por parte del observatorio de seguimiento del nuevo baremo.

5. Ha habido una mejora significativa en la claridad terminológica y adecuación a los términos manejados en la literatura médica actual en la redacción del nuevo baremo. Sin embargo, sería conveniente que las fuentes de validación de sus estimaciones fuesen especificadas y que el baremo se acompañase de una guía oficial de condiciones técnicas en las cuales las medidas objetivas debieran ser tomadas.

6. La consistencia interna o validez en sus sistemas de asignación de grados de gravedad ha mejorado frente al baremo precedente. Sin embargo, sigue presentando en los capítulos de raquis y extremidades algunos defectos severos que exigirían una revisión crítica por parte del observatorio de seguimiento del nuevo baremo.

7. El nuevo baremo ha mejorado su congruencia con otros baremos ya existentes y aceptados por la comunidad científica, como las tablas AMA, pero presenta con estos diferencias significativas que afectan tanto a sus principios metodológicos como a los resultados finales de valoración de menoscabo obtenidos. El nuevo baremo presenta poca congruencia con algunos estudios que han valorado la eficacia relativa de los métodos de cuantificación como predictores de un menoscabo funcional proporcionado y con las recomendaciones de los comités de expertos disponibles.

8. Las conclusiones anteriores hacen que posiblemente la eficacia del baremo de la Ley 34/2003 como baremo de cuantificación de daños funcionales (menoscabo físico y psíquico) sea limitada. Los resultados de gravedad relativa obtenidos posiblemente tengan un fundamento médico escaso y respondan pobremente a un principio de proporcionalidad. La reproducibilidad de los resultados posiblemente sea escasa. Una revisión crítica por parte del observatorio de seguimiento del baremo sería conveniente para asegurar la elaboración de un nuevo baremo de menoscabo funcional más ajustado al actual estado de las ciencias forenses en España. 
Reflexiones sobre la Ley 34/2003 (3). Valoración de los capítulos relativos a raquis, extremidades superiores e inferiores.

\begin{tabular}{|c|c|c|c|c|c|c|c|c|}
\hline & Ley $30 / 95$ & & Ley $34 / 03$ & & RD 1971/99 & & Buckup & Kapandji (*) \\
\hline $\begin{array}{l}\text { Arcos } \\
\text { normales de } \\
\text { movilidad } \\
\text { Cervical }\end{array}$ & $\begin{array}{l}\text { Flexo-extensión } \\
(40 / 75) 115^{\circ} \\
\text { Inclinación dcha-izda } \\
(30-45 / 30-45) 60-90^{\circ} \\
\text { Rotación dcha-izda } \\
(50 / 50) 100^{\circ}\end{array}$ & & No especifica & & $\begin{array}{l}\text { Flexo-extensión } \\
(50 / 60) 110^{\circ} \\
\\
\text { Inclinación } \\
(45 / 45) 90^{\circ} \\
\text { Rotación axial } \\
(80 / 80) 160^{\circ}\end{array}$ & $\begin{array}{l}12 \\
8\end{array}$ & $\begin{array}{l}\text { Flexión-extensión } \\
\left(35-45 / 35-45^{\circ}\right) \\
70-90^{\circ} \\
\text { Inclinación } \\
\left(45 / 45^{\circ}\right) 90^{\circ} \\
\\
\text { Rotación } \\
\left(60-80 / 60-80^{\circ}\right) \\
120-160^{\circ} \\
\end{array}$ & $\begin{array}{l}\text { Flexión-extensión } \\
\left(40 / 75^{\circ}\right) 115^{\circ} \\
\\
\text { Inclinación } \\
\left(35-45 / 35-45^{\circ}\right) 70-90^{\circ} \\
\text { Rotación axial } \\
\left(45-50 / 45-50^{\circ}\right) 90-100^{\circ}\end{array}$ \\
\hline $\begin{array}{l}\text { Limitación de } \\
\text { movilidad } \\
\text { cervical }\end{array}$ & & $5-15$ & & $5-15$ & $\begin{array}{l}\text { Suma de limitación } \\
\text { de movilidad } \\
\text { Anquilosis funcional } \\
\text { Anquilosis no } \\
\text { funcional (máxima) }\end{array}$ & $\begin{array}{l}28 \\
29 \\
82\end{array}$ & & \\
\hline $\begin{array}{l}\text { Arcos } \\
\text { normales de } \\
\text { movilidad } \\
\text { toraco- } \\
\text { lumbar }\end{array}$ & $\begin{array}{l}\text { Dorso-lumbar: } \\
\text { Flexión } 150^{\circ} \\
\text { Extensión } 60^{\circ} \\
\text { Inclinación dcha-izda } \\
\left(20 / 20^{\circ}\right) 40^{\circ} \\
\text { Rotación dcha-izda } \\
(35 / 35) 70^{\circ}\end{array}$ & & No especifica & & $\begin{array}{l}\text { Dorsal: } \\
\text { Flexión } 50^{\circ} \\
\text { Extensión } 0^{\circ} \\
\text { Rotación }(30 / 30) 60^{\circ} \\
\text { Lumbo-sacro ("*): } \\
\text { Flexión } 90^{\circ} \\
\text { Extensión } 30^{\circ} \\
\text { Inclinación lateral } \\
(25 / 25) 50^{\circ}\end{array}$ & & $\begin{array}{l}\text { Dorso-lumbar: } \\
\text { Flexión DDS } \\
\text { Extensión } 30^{\circ} \\
\text { Inclinación lateral } \\
(30-40 / 30-40) 80^{\circ} \\
\text { Rotación axial } \\
(30 / 30) 60^{\circ}\end{array}$ & $\begin{array}{l}\text { Dorso-lumbar: } \\
\text { Flexión } 105^{\circ} \\
\text { Extensión } 35^{\circ} \\
\\
\text { Dorsal: } \\
\text { Inclinación lateral } \\
(20 / 20) 40^{\circ} \\
\text { Rotación axial } \\
(35 / 35) 70^{\circ} \\
\text { Lumbar: } \\
\text { Flexión } 60^{\circ} \\
\text { Extensión } 35^{\circ} \\
\text { Inclinación lateral } \\
(20 / 20) 40^{\circ} \\
\text { Rotación axial } \\
(5 / 5) 10^{\circ}\end{array}$ \\
\hline $\begin{array}{l}\text { Limitación de } \\
\text { movilidad } \\
\text { dorso-lumbar }\end{array}$ & $\begin{array}{l}\text { Rigidez dorsal o } \\
\text { lumbar con ligera } \\
\text { dificultad en los } \\
\text { movimientos por } \\
\text { fracturas vertebrales } \\
\text { (<30\% de } \\
\text { disminución de } \\
\text { movilidad) } \\
\text { Rigidez dorsal o } \\
\text { lumbar con } \\
\text { importante dificultad } \\
\text { en los movimientos } \\
\text { por fracturas } \\
\text { vertebrales ( }>30 \% \text { de } \\
\text { disminución de } \\
\text { movilidad) } \\
\end{array}$ & $\begin{array}{l}2-10 \\
10-25 \\
\end{array}$ & $\begin{array}{l}\text { Limitación de } \\
\text { movilidad } \\
\text { toraco-lumbar }\end{array}$ & $2-25$ & $\begin{array}{l}\text { Suma de limitación } \\
\text { movilidad } \\
\text { Dorsal } \\
\text { Lumbar } \\
\text { Anquilosis Dorsal } \\
\text { Funcional } \\
\begin{array}{l}\text { No funcional } \\
\text { (máxima) }\end{array} \\
\text { Anquilosis Lumbar } \\
\text { Funcional } \\
\begin{array}{l}\text { No Funcional } \\
\text { (máxima) }\end{array}\end{array}$ & $\begin{array}{l}28 \\
82\end{array}$ & & \\
\hline $\begin{array}{l}\text { Arco normal } \\
\text { de movilidad } \\
\text { global del } \\
\text { Raquis }\end{array}$ & & & & & & & & $\begin{array}{l}\text { Flexión total } 110^{\circ} \\
\text { Extensión total } 140^{\circ} \\
\text { Inclinación total } \\
(75-80 / 75-80) \\
150-160^{\circ} \\
\text { Rotación axial total } \\
(90-95 / 90-95) \\
180-190^{\circ}\end{array}$ \\
\hline
\end{tabular}

Tabla 1. Columna vertebral. Arcos de movimiento.

Se comparan en esta tabla los datos derivados del estudio de las tabla de la Ley 30/1995 [2], de la Ley 34/2003 [1], del RD 1971/1999 (versión española de las tablas AMA) [10] y de los rangos de normalidad considerados en el texto de Buckup $K$ [24] y el de Kapandji AI [15]. No se incluyen los arcos de movimiento considerados normales en el Proyecto de baremo europeo [13] al no reflejarse en su redacción.

* De acuerdo con el texto de Kapandji Al, las medidas indicadas corresponden con los máximos balances articulares medidos en estudios radiográficos. El autor especifica que las medidas son superiores a las que pueden estimarse en la exploración física convencional de sujetos normales.

** Según la redacción del RD 1971/1999, al menos un 50\% del arco de movimiento de flexo-extensión de la columna lumbosacra corresponde al movimiento del segmento de sacro y caderas. 


\begin{tabular}{|c|c|c|c|c|}
\hline & $\stackrel{9}{\mathrm{v}}$ & 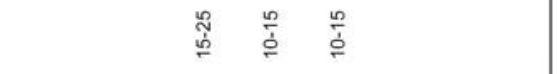 & 足 & 항 \& \\
\hline & 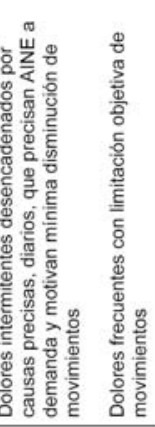 & 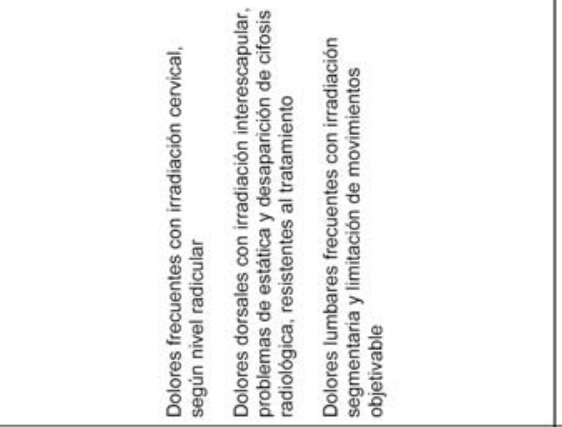 & 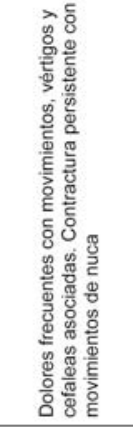 & 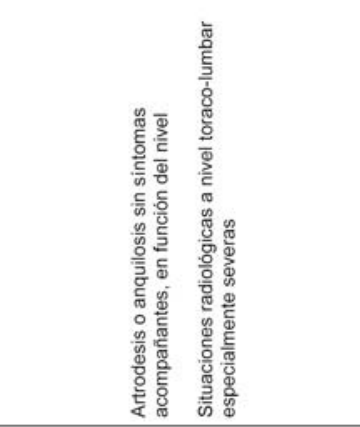 \\
\hline & $\circ \infty$ & 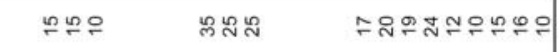 & 0 in & to: NMS SR \\
\hline \multirow[t]{2}{*}{ 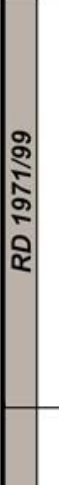 } & 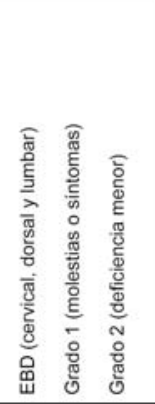 & 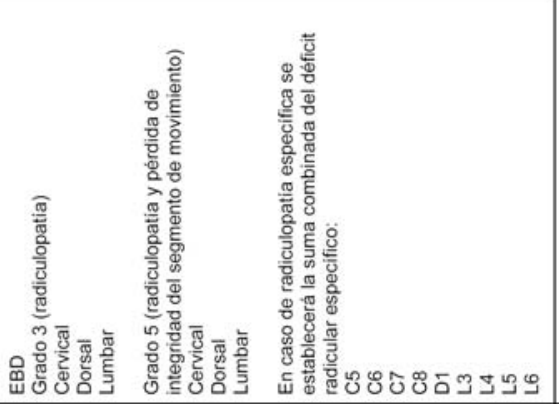 & 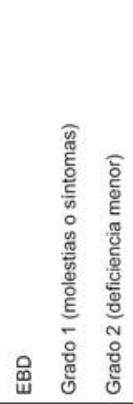 & 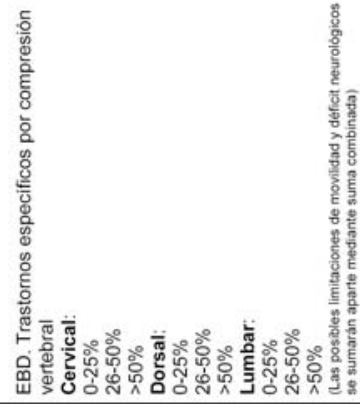 \\
\hline & $\stackrel{\varphi}{\longrightarrow}$ & 号 & $\stackrel{\infty}{-}$ & $\stackrel{\circ}{\stackrel{1}{i}}$ \\
\hline \multirow[t]{2}{*}{ בֶ๊ } & 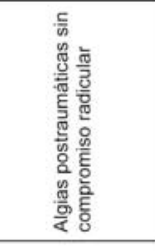 & 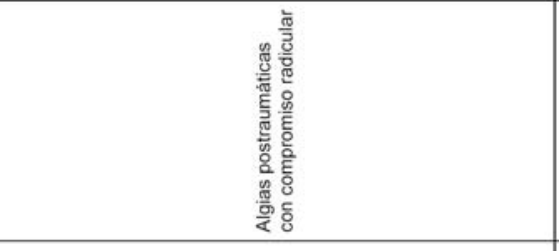 & 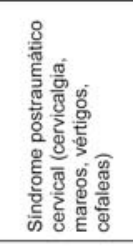 & 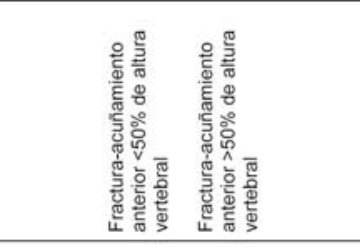 \\
\hline & $\stackrel{\varphi}{=} \stackrel{\sim}{\mathcal{N}}$ & 号䓂菅 & $\stackrel{\Phi}{-}$ & $\frac{\circ}{\dot{N}} \quad \frac{n}{\dot{d}}$ \\
\hline \multirow[t]{2}{*}{ } & 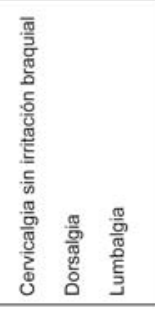 & 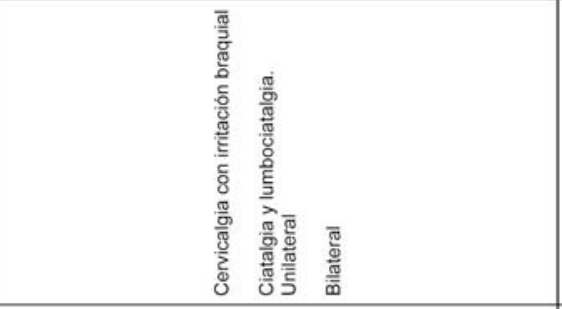 & 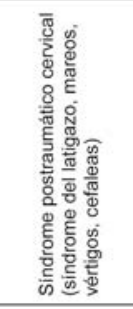 & 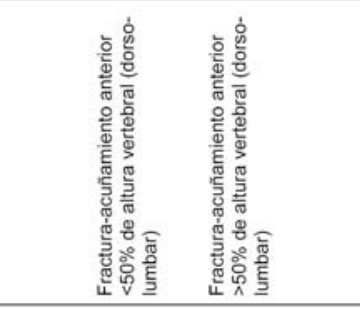 \\
\hline & 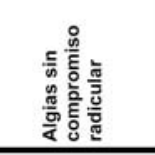 & 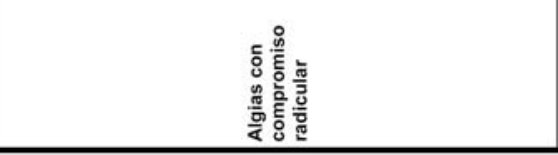 & 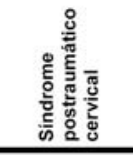 & 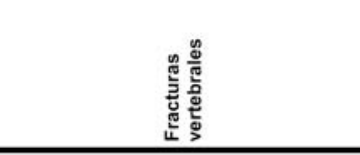 \\
\hline
\end{tabular}




\begin{tabular}{|c|c|}
\hline 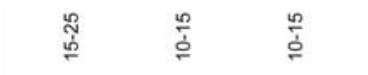 & $\stackrel{\mathscr{q}}{v}$ \\
\hline 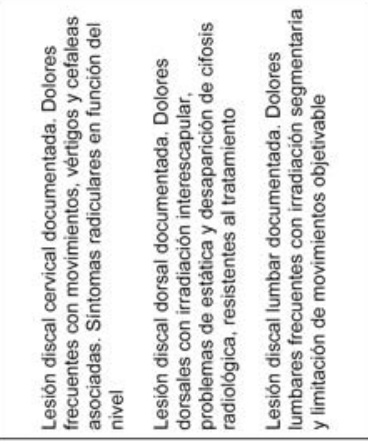 & 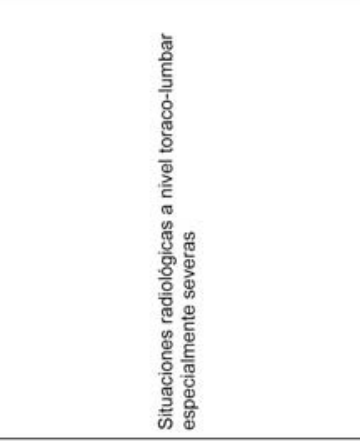 \\
\hline$a \infty \div$ & 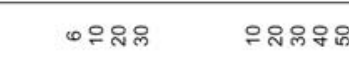 \\
\hline 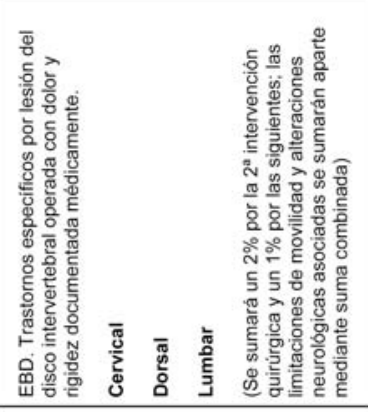 & 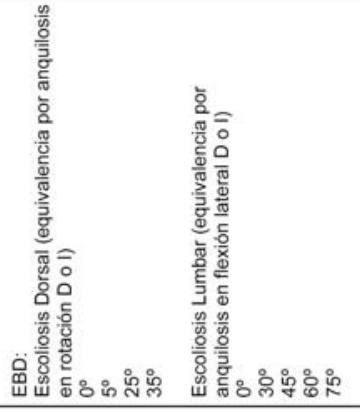 \\
\hline$\stackrel{\varphi}{\longleftarrow}$ & 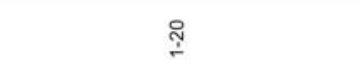 \\
\hline 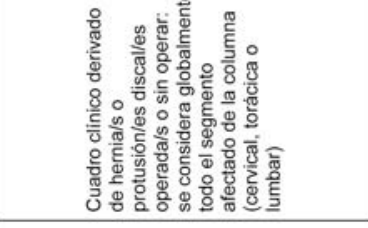 & 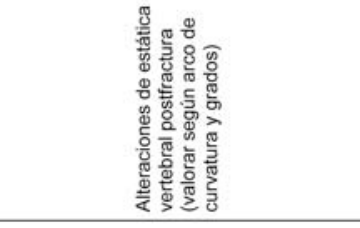 \\
\hline$\stackrel{0}{\omega^{\circ}}$ & 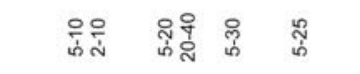 \\
\hline 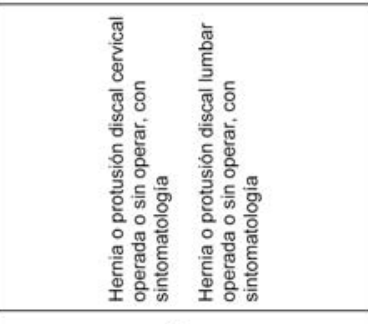 & 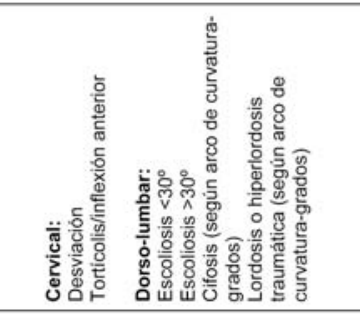 \\
\hline 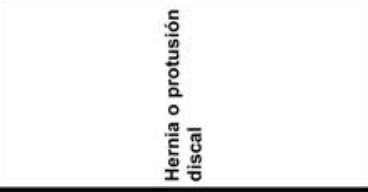 & 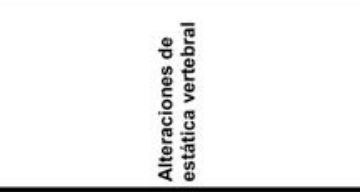 \\
\hline
\end{tabular}




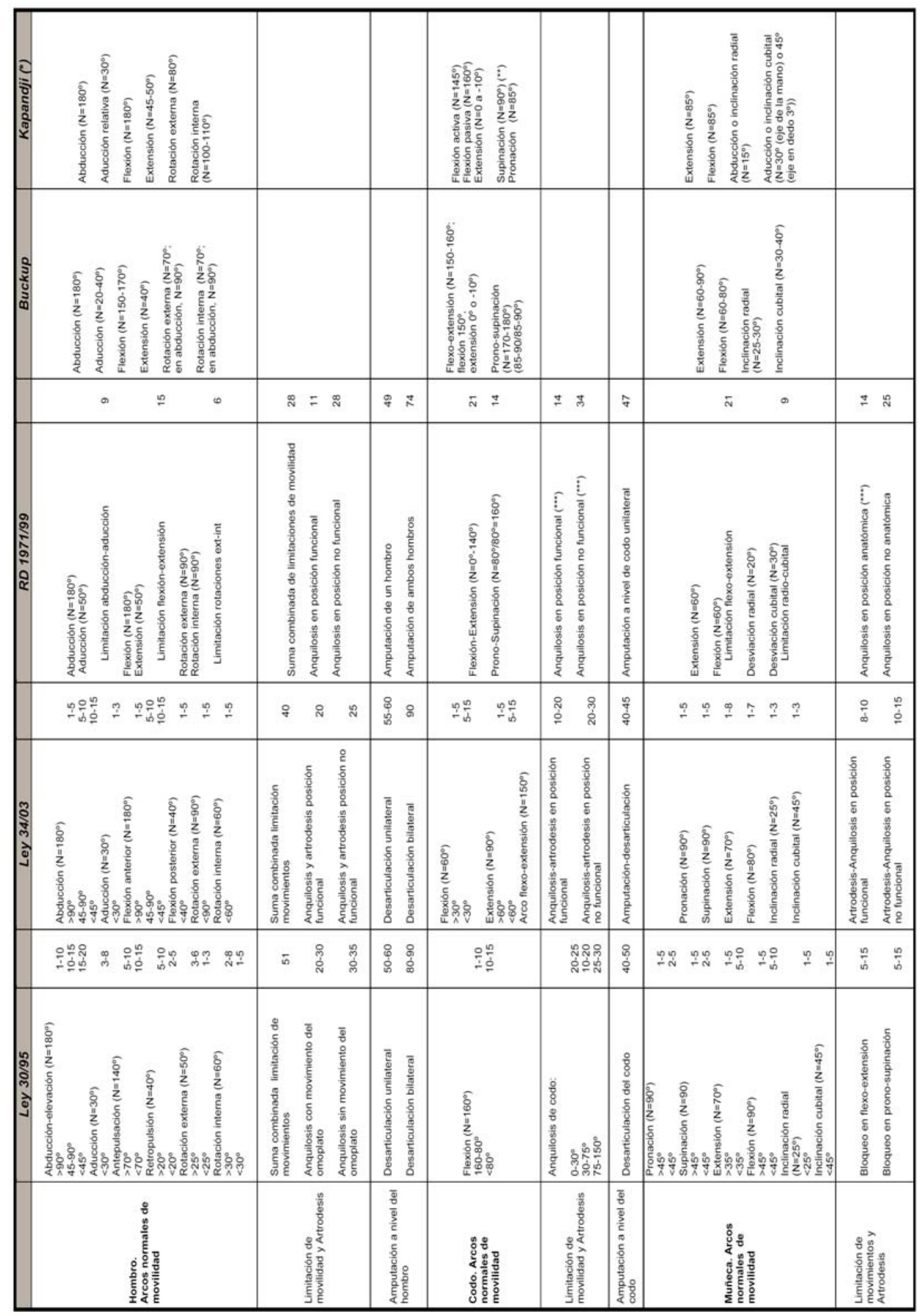



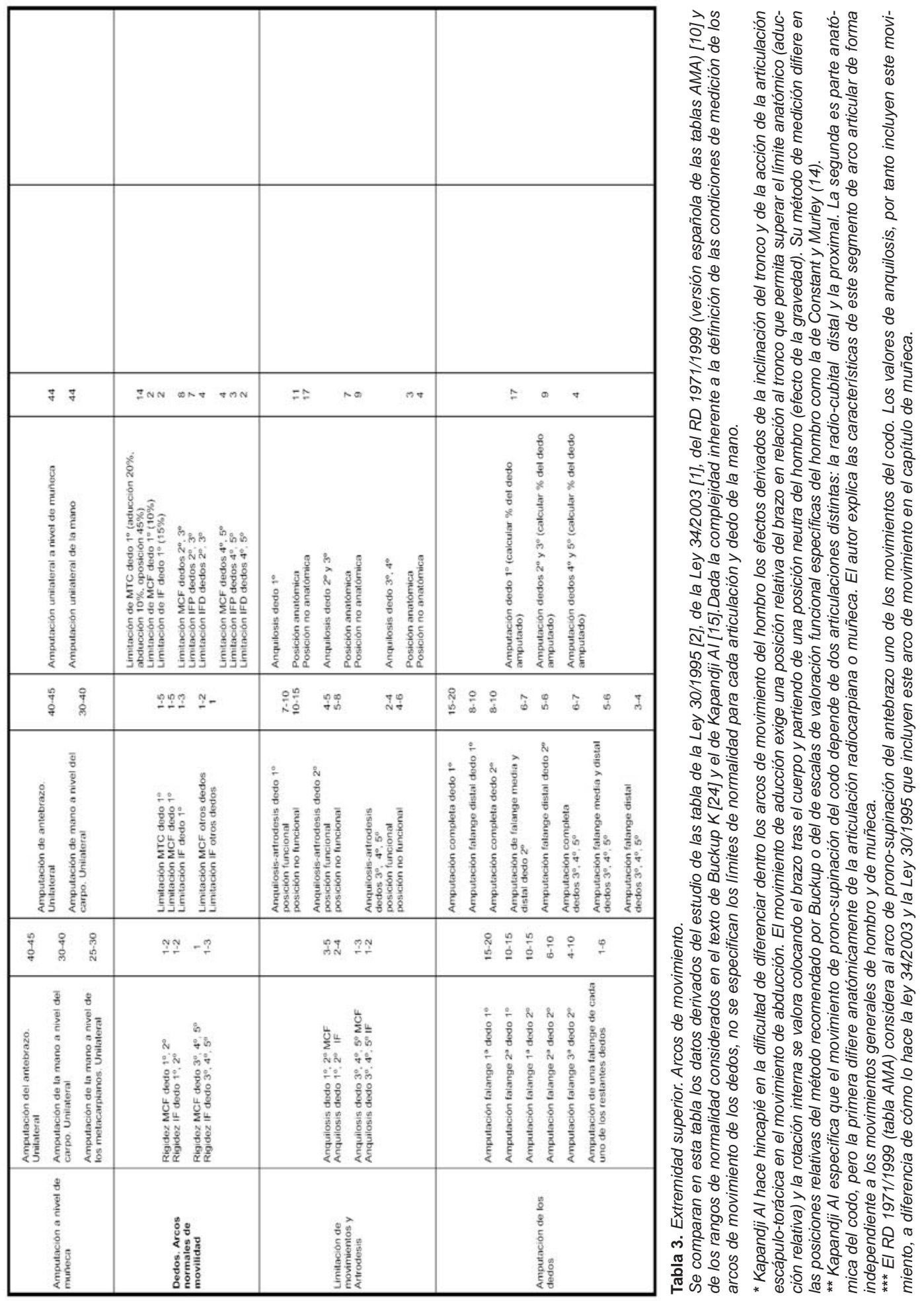
Reflexiones sobre la Ley 34/2003 (3). Valoración de los capítulos relativos a raquis, extremidades superiores e inferiores.

\begin{tabular}{|c|c|c|c|c|c|c|c|c|}
\hline & Ley 30/95 & & Ley $34 / 03$ & & RD 1971/99 & & Buckup & Kapandji \\
\hline $\begin{array}{l}\text { Cadera. } \\
\text { Arcos normales } \\
\text { de movilidad }\end{array}$ & $\begin{array}{l}\text { Flexión }\left(\mathrm{N}=120^{\circ}\right) \\
>90^{\circ} \\
<90^{\circ} \\
\text { Extensión }\left(\mathrm{N}=20^{\circ}\right) \\
<20^{\circ} \\
\text { Abducción }\left(\mathrm{N}=60^{\circ}\right) \\
>30^{\circ} \\
<30^{\circ} \\
\text { Rotación interna }\left(\mathrm{N}=30^{\circ}\right) \\
<30^{\circ} \\
\\
\text { Rotación externa } \\
\left(\mathrm{N}=60^{\circ}\right) \\
\left.>30^{\circ}\right) \\
<30^{\circ}\end{array}$ & $\begin{array}{c}2-10 \\
10-15 \\
2-10 \\
\\
1.5 \\
5-10 \\
1.5\end{array}$ & $\begin{array}{l}\text { Flexión }\left(\mathrm{N}=120^{\circ}\right) \\
>90^{\circ} \\
45-90^{\circ} \\
<45^{\circ} \\
\\
\text { Extensión }\left(\mathrm{N}=20^{\circ}\right) \\
<20^{\circ} \\
\\
\text { Abducción }\left(\mathrm{N}=60^{\circ}\right) \\
>30^{\circ} \\
<30^{\circ} \\
\\
\text { Rotación interna }\left(\mathrm{N}=30^{\circ}\right) \\
<30^{\circ} \\
\\
\text { Rotación externa } \\
\left(\mathrm{N}=60^{\circ}\right) \\
>30^{\circ} \\
<30^{\circ}\end{array}$ & $\begin{array}{c}1-5 \\
5-10 \\
10-15 \\
\\
1-5 \\
\\
\\
1-3 \\
3-6 \\
\\
1-3\end{array}$ & $\begin{array}{l}\text { Flexión }\left(\mathrm{N}=100^{\circ}\right) \\
\text { Extensión }\left(\mathrm{N}=30^{\circ}\right) \\
\text { Abducción }\left(\mathrm{N}=40^{\circ}\right) \\
\text { Abducción }\left(\mathrm{N}=20^{\circ}\right) \\
\text { Rotación interna }\left(\mathrm{N}=30^{\circ}\right) \\
\begin{array}{l}\text { Rotación externa } \\
\left(\mathrm{N}=50^{\circ}\right)\end{array}\end{array}$ & 8 & $\begin{array}{l}\text { Flexión }\left(\mathrm{N}=130-140^{\circ}\right) \\
\text { Extensión }\left(\mathrm{N}=0^{\circ}\right) \\
\text { Abducción }\left(\mathrm{N}=30-45^{\circ}\right) \\
\text { Aducción }\left(\mathrm{N}=20-30^{\circ}\right) \\
\begin{array}{l}\text { Rotación interna } \\
\left(\mathrm{N}=30-40^{\circ}\right)\end{array} \\
\begin{array}{l}\text { Rotación externa } \\
\left(\mathrm{N}=40-50^{\circ}\right)\end{array}\end{array}$ & 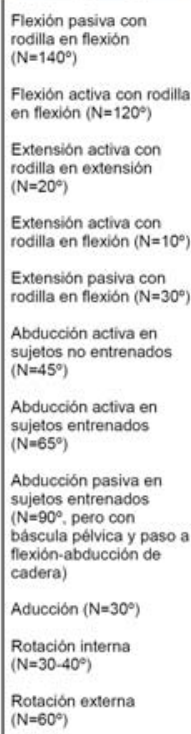 \\
\hline $\begin{array}{l}\text { Limitación de } \\
\text { movilidad y } \\
\text { Artrodesis }\end{array}$ & $\begin{array}{l}\text { Suma combinada } \\
\text { limitación movimientos } \\
\text { Anquilosis en posición } \\
\text { favorable de una cadera } \\
\text { Anquilosis en posición } \\
\text { no favorable }\end{array}$ & $\begin{array}{c}42 \\
20-25 \\
25-40\end{array}$ & $\begin{array}{l}\text { Suma combinada } \\
\text { limitación movimientos } \\
\text { Anquilosis y artrodesis } \\
\text { posición funcional } \\
\text { Anquilosis y artrodesis } \\
\text { posición no funcional }\end{array}$ & $\begin{array}{c}31 \\
25 \\
25-35\end{array}$ & $\begin{array}{l}\text { Suma combinada de } \\
\text { limitaciones de movilidad } \\
\text { Anquilosis en posición } \\
\text { optima } \\
\text { Anquilosis en posición } \\
\text { pésima }\end{array}$ & 20 & & \\
\hline $\begin{array}{l}\text { Amputación a } \\
\text { nivel de la cadera }\end{array}$ & $\begin{array}{l}\text { Amputación unilateral } \\
\text { Amputación bilateral }\end{array}$ & $\begin{array}{l}60-70 \\
90-95\end{array}$ & $\begin{array}{l}\text { Desarticulación } \\
\text { unilateral } \\
\text { Desarticulación bilateral }\end{array}$ & $\begin{array}{l}60-70 \\
90-95\end{array}$ & $\begin{array}{l}\text { Amputación de una } \\
\text { cadera }\end{array}$ & 40 & & \\
\hline $\begin{array}{l}\text { Rodilla. Arcos } \\
\text { normales de } \\
\text { movilidad }\end{array}$ & $\begin{array}{l}\text { Flexión }\left(\mathrm{N}=135^{\circ}\right) \\
>90^{\circ} \\
<90^{\circ} \\
\text { Limitación de extensión } \\
\text { de rodilla en los últimos } \\
20^{\circ}\end{array}$ & \begin{tabular}{c|}
$1-10$ \\
$10-20$ \\
$1-15$
\end{tabular} & $\begin{array}{l}\text { Flexión }\left(\mathrm{N}=135^{\circ}\right) \\
>990^{\circ} \\
90-45^{\circ} \\
<45^{\circ} \\
\text { Extension }\left(\mathrm{N}=?^{\circ}\right) \\
\text { Mueve }>10^{\circ} \\
\text { Mueve }<10^{\circ}\end{array}$ & $\begin{array}{c}1.5 \\
5-10 \\
10-15 \\
\\
1.5 \\
5-15\end{array}$ & $\begin{array}{l}\text { Flexión }\left(\mathrm{N}=140^{\circ}\right) \\
\text { Extensión }\left(\mathrm{N}=0^{\circ}\right) \\
\text { Contractura en flexo } \\
\text { de }+20^{\circ}\end{array}$ & 14 & $\begin{array}{l}\text { Flexión }\left(\mathrm{N}=120-150^{\circ}\right) \\
\text { Extensión }\left(\mathrm{N}=5-10^{\circ}\right) \\
\text { Rotación interna con } \\
\text { rocilla en flexión de } 90^{\circ} \\
\left(\mathrm{N}=10^{\circ}\right) \\
\text { Rotación externa con } \\
\text { rodilla en flexión de } 90^{\circ} \\
\left(\mathrm{N}=25^{\circ}\right)\end{array}$ & $\begin{array}{l}\text { Flexión activa con } \\
\text { cadera en flexión } \\
\left(\mathrm{N}=140^{\circ}\right) \\
\text { Flexión activa con } \\
\text { cadera en extensión } \\
\left(\mathrm{N}=120^{\circ}\right) \\
\text { Flexión pasiva con } \\
\text { cadera en flexión } \\
\left(\mathrm{N}=160^{\circ}\right) \\
\begin{array}{l}\text { Extension pasiva } \\
\left(\mathrm{N}=5-10^{\circ}\right)\end{array}\end{array}$ \\
\hline $\begin{array}{l}\text { Limitación de } \\
\text { movilidad y } \\
\text { Artrodesis }\end{array}$ & $\begin{array}{l}\text { Anquilosis de rodilla en } \\
\text { extension (posición de } \\
\text { función) unilateral }\end{array}$ & $20-30$ & $\begin{array}{l}\text { Anquilosis-artrodesis en } \\
\text { posición funcional } \\
\text { Anquilosis-artrodesis en } \\
\text { posición no funcional }\end{array}$ & 20 & $\begin{array}{l}\text { Anquilosis en posición } \\
\text { optima } \\
\text { Anquilosis en posición } \\
\text { pésima }\end{array}$ & $\begin{array}{l}29 \\
38\end{array}$ & & \\
\hline $\begin{array}{l}\text { Amputación a } \\
\text { nivel de rodilla }\end{array}$ & $\begin{array}{l}\text { Amputación a nivel de } \\
\text { rodilla }\end{array}$ & $55-60$ & $\begin{array}{l}\text { Amputación a nivel } \\
\text { diafisario de fémur o en } \\
\text { rodilla }\end{array}$ & $50-60$ & $\begin{array}{l}\text { Amputación a nivel de } \\
\text { rodilia unilateral }\end{array}$ & 32 & & \\
\hline $\begin{array}{l}\text { Articulación } \\
\text { tibio-tarsiana y } \\
\text { tarso y pie ("). } \\
\text { Arcos normales } \\
\text { de movilidad }\end{array}$ & 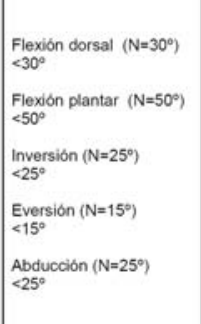 & $\begin{array}{l}1-5 \\
1-10 \\
1-5 \\
1-5 \\
1-5\end{array}$ & 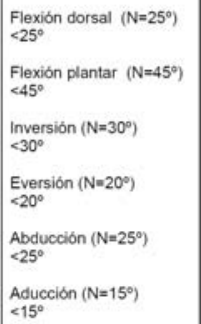 & $\begin{array}{l}1.5 \\
1-7 \\
1.3 \\
1.3 \\
1.3 \\
1.3\end{array}$ & $\begin{array}{l}\text { Flexión dorsal }\left(\mathrm{N}=20^{\circ}\right) \\
\text { Flexión plantar }\left(\mathrm{N}=40^{\circ}\right) \\
\text { Inversión }\left(\mathrm{N}=30^{\circ}\right) \\
\text { Eversión }\left(\mathrm{N}=20^{\circ}\right) \\
\text { Abducción }\left(\mathrm{N}=25^{\circ}\right) \\
\text { Aducción }\left(\mathrm{N}=20^{\circ}\right)\end{array}$ & $\begin{array}{l}3 \\
12 \\
2 \\
1 \\
\text { ¿? } \\
\text { ¿? }\end{array}$ & $\begin{array}{l}\text { Flexión dorsal }\left(\mathrm{N}=30^{\circ}\right) \\
\text { Flexión plantar }\left(\mathrm{N}=50^{\circ}\right) \\
\text { Inversión }\left(\mathrm{N}=20^{\circ}\right) \\
\text { Eversión }\left(\mathrm{N}=16^{\circ}\right) \\
\text { Pronación }\left(\mathrm{N}=15^{\circ}\right) \\
\text { Supinación }\left(\mathrm{N}=35^{\circ}\right)\end{array}$ & $\begin{array}{l}\text { Flexion (dorsal) } \\
\left(\mathrm{N}=20-30^{\circ}\right) \\
\text { Extensión } \\
\text { (flexión plantar) } \\
\left(\mathrm{N}=30-50^{\circ}\right) \\
(\cdot-)\end{array}$ \\
\hline
\end{tabular}




\begin{tabular}{|c|c|c|c|c|c|c|c|}
\hline $\begin{array}{l}\text { Limitacion de } \\
\text { movimientosy } \\
\text { Aftrodesis }\end{array}$ & $\begin{array}{l}\text { Suma limitación de } \\
\text { flexion plantar-dorsal } \\
\text { Suma de limitación de } \\
\text { movimientos globales } \\
\text { Artrodesis } \\
\text { subastragalina } \\
\text { Triple artrodesis }\end{array}$ & \begin{tabular}{l|}
15 \\
28 \\
$8-12$ \\
$8-12$
\end{tabular} & 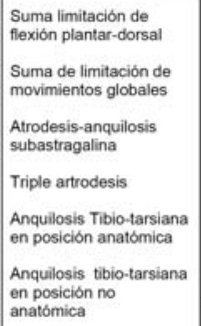 & \begin{tabular}{|c|}
12 \\
22 \\
$5-8$ \\
10 \\
12 \\
$12-20$
\end{tabular} & 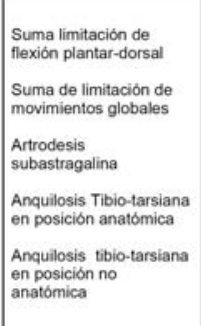 & $\begin{array}{l}15 \\
2 ? \\
21 \\
4 \\
25\end{array}$ & \\
\hline $\begin{array}{l}\text { Amputación tibio- } \\
\text { tarsiana }\end{array}$ & \begin{tabular}{|l} 
Amputación tibio- \\
tarsiana uninateral. \\
Unilateral \\
Amputacion a nivel de \\
tarso. Unilateral \\
Amputacion de la mano \\
a nivel de los la \\
metacarpianos. \\
Unilateral
\end{tabular} & $\begin{array}{l}30-40 \\
20-30\end{array}$ & $\begin{array}{l}\text { Amputación a nivel de } \\
\text { tarso o tibiotarsiana. } \\
\text { Unilateral } \\
\text { Amputación a nivel de } \\
\text { metatarso y tarso. } \\
\text { Unilateral }\end{array}$ & $\begin{array}{l}30-40 \\
15-30\end{array}$ & $\begin{array}{l}\text { Amputación unilateral a } \\
\text { nivel de tarso }\end{array}$ & $16-18$ & \\
\hline $\begin{array}{l}\text { Deformidades } \\
\text { del pie }\end{array}$ & \begin{tabular}{|l} 
Pie plano \\
Pie cavo \\
Pie talo \\
Pie equino \\
Pie valgo \\
Pie varo \\
Pie zambo
\end{tabular} & \begin{tabular}{|l|}
$5-10$ \\
$5-10$ \\
$7-12$ \\
$8-15$ \\
$5-10$ \\
$5-10$ \\
$5-20$
\end{tabular} & $\begin{array}{l}\text { Deformidades } \\
\text { postraumáticicas } \\
\text { (valgo, varo, etc) }\end{array}$ & $1-10$ & \begin{tabular}{|l} 
Deformidades del \\
retropie \\
Deformidades del \\
mesopie \\
Deformidades del \\
antepie
\end{tabular} & 8 & \\
\hline $\begin{array}{l}\text { Dedos del pie. } \\
\text { Amputaciones }\end{array}$ & $\begin{array}{l}\text { Amputación dedo } 1^{\circ} \\
\text { Amputación resto dedos }\end{array}$ & $\begin{array}{c}5.10 \\
1.6\end{array}$ & $\begin{array}{l}\text { Amputación dedo } 1^{\circ} \\
\text { Amputación dedo } 1^{\circ} \\
\text { falange distal } \\
\text { Amputación resto dedos } \\
\begin{array}{l}\text { Amputacion resto dedos } \\
\text { falanges } 2^{\circ} \text { y } 3^{\circ}\end{array}\end{array}$ & $\begin{array}{l}10 \\
3 \\
3 \\
1\end{array}$ & $\begin{array}{l}\text { Amputación dedo } 1^{\circ} \\
\text { MTF } \\
\text { Amputación dedo } 1^{\circ} \mathrm{IF} \\
\text { Amputación dedos } 2^{\circ} \text { a } \\
5^{\circ} \mathrm{MTF} \\
\text { Amputación todos los } \\
\text { dedos en MTF }\end{array}$ & 2 & \\
\hline
\end{tabular}

Tabla 4. Extremidad superior. Arcos de movimiento.

Se comparan en esta tabla los datos derivados del estudio de las tabla de la Ley 30/1995 [2], de la Ley 34/2003 [1], del RD 1971/1999 (versión española de las tablas AMA) [10] y de los rangos de normalidad considerados en el texto de Buckup K [24] y el de Kapandji Al [15].

* La redacción de las leyes 34/2003 y 30/1995 es contradictoria en la asignación del segmento anatómico al que adscribir los movimientos indicados. La redacción actual es congruente con las recomendaciones AMA y las estimaciones de textos de fisiología articular. Se ha decidido unir las categorías de arcos de movimiento de las distintas articulaciones del tobillo y tarso para facilitar la comprensión visual de la comparación entre baremos

** Kapandji Al no especificar claramente los límites máximos admisibles para los distintos segmentos de movilidad de las articulaciones de tarso y tobillo. 


\section{BIBLIOGRAFÍA:}

I. Ley 34/2003 de 4 de noviembre de modificación y adaptación a la normativa comunitaria de la legislación de seguros privados. BOE 5 II-2003.

http://www.justizia.net/docuteca/ficheros.asp?intcodigo $=|58| \& \mid d$ Doc $=S P$

2. Ley 30/1995 de 8 de noviembre de ordenación y supervisión de seguros privados. BOE. 1995; vol de 9 de noviembre: 32480-32567. http://www.boe.es/

3. Vicente Domingo E. Los daños corporales: tipología y valoración. JM Bosch Editor SA. 1994.

4. Miralles RC. Valoración del daño corporal en el aparato locomotor. Masson. 2001.

5. Borobia C. Análisis del anteproyecto de la reforma de la tabla VI de la Ley 30/1995. VII Jornadas Catalanas de Actualización en Medicina Forense. Barcelona 12-6-2003.

6. Alonso J. La nueva tabla VI. Presentación y análisis. Principales mejoras y diferencias. Jornada sobre "baremo de daños corporales. Nueva tabla VI", Bilbao 26-I-2004. http://www.justizia.net/ivml 7. Portero G. Valoración de la tabla psiquiátrica. Comentarios y críticas a la Ley 34/2003. Jornada sobre "baremo de daños corporales. Nueva tabla VI", Bilbao 26-I-2004. http://www.justizia.net/ivml 8. Garamendi PM. Cambios realizados en la tabla VI de la Ley 30/1995 por la Ley 34/2003. Extremidades superiores e inferiores. Jornada sobre "baremo de daños corporales. Nueva tabla VI", Bilbao 26-I-2004. http://www.justizia.net/ivml

9. Garamendi PM. Reflexiones sobre la Ley 34/2003 (I). Consideraciones generales sobre las tablas de indemnización de daños personales. Cuad Med For 2004;36:19-28.

10. Real Decreto 1971/1999, de 23 de diciembre de procedimiento para el reconocimiento, declaración y calificación del grado de minusvalía. BOE. 2000; 26 de enero: 3317-3410. http://www.boe.es II. New South Wales Motor Accidents Authority Guidelines for the Assessment of Permanent Impairment of a Person injured as a result of a motor accident. MAA Guidelines, March 2000.
12. Melennec, L Evaluation du handicap et du dommage corporel. Barème international des invalidités. Masson 1991.

13. Guide barème européen d'évaluation des atteintes à l' intégrité physique et psychique. Groupe Rothley. 25-mai-2003.

14. Constant CR, Murley AHG. A clinical method of functional assessment of the shoulder. Clin Orthop Rel Res 1987; 214:160-4

15. Kapandji Al. Fisiología articular. Editorial Médica Panamericana. 2001.

16. Pynset PB, Fairbank JCT, Carr AJ. Medición de resultados en Traumatología. Masson. 1997.

17. Harper JD. Determining foot and ankle impairments by the AMA fifth edition guides. Foot Ankle Clin 2002; 7(2): 291-303

18. Zuberbier OA, Hunt DG, Kozlowski AJ, Berkowitz J, Schultz IZ, Crook JM, Milner RA. Commentary on the American Medical Association guides' lumbar impairment validity checks. Spine 200I; 26(24): 2735-7.

19. Nattrass CL, Nitschke JE, Disler PB, Chou MJ, Ooi KT. Lumbar spine range of motion as a measure of physical and functional impairment: an investigation of validity. Clin Rehabil 1999; 13(3): 2II-8.

20. Lowery WD Jr, Horn TJ, Boden SD, Wiesel SW. Impairment evaluation based on spinal range of motion in normal subjects. J Spinal Disord 1992; 5(4): 398-402.

2I. Rondinelli RD, Dunn W, Hassanein KM, Keesling CA, Meredith SC, Schulz TL, Lawrence NJ. A simulation of hand impairments: effects on upper extremity function and implications toward medical impairment rating and disability determination. Arch Phys Med Rehabil 1997; 78(I2): I358-63.

22. International Statistical Classification of Diseases and Related Health Problems (ICD-I0), 1989 Revision, Geneva, World Health Organization, 1992.

23. Peterson F, Kendall E, Geise P. Kendall 's. Músculos: pruebas, funciones y dolor postural. $4^{a}$ edición. Marban, 2000.

24. Buckup K. Pruebas clínicas para patología ósea, articular y muscular. Exploración, signos y síntomas. Masson. 2000. 